2013

\title{
Unusually large spin polarization and magnetoresistance in a FeMg8-FeMg8 superatomic dimer
}

Lin Zhu

Virginia Commonwealth University, Huazhong University of Science and Technology

Meichun Qian

Virginia Commonwealth University,mqian@vcu.edu

Shiv N. Khanna

Virginia Commonwealth University, snkhanna@vcu.edu

Follow this and additional works at: http://scholarscompass.vcu.edu/phys_pubs

Part of the Physics Commons

Zhu, L., Qian, M., \& Khanna, S. N. Unusually large spin polarization and magnetoresistance in a FeMg8-FeMg8 superatomic dimer. The Journal of Chemical Physics, 139, 064306 (2013). C 2013 Author(s). All article content, except where otherwise noted, is licensed under a Creative Commons Attribution 3.0 Unported License.

\section{Downloaded from}

http://scholarscompass.vcu.edu/phys_pubs/112

This Article is brought to you for free and open access by the Dept. of Physics at VCU Scholars Compass. It has been accepted for inclusion in Physics Publications by an authorized administrator of VCU Scholars Compass. For more information, please contact libcompass@vcu.edu. 


\title{
Unusually large spin polarization and magnetoresistance in a $\mathrm{FeMg}_{8}-\mathrm{FeMg}_{8}$ superatomic dimer
}

\author{
Lin Zhu, ${ }^{1,2}$ Meichun Qian, ${ }^{1}$ and Shiv N. Khanna ${ }^{1, a)}$ \\ ${ }^{1}$ Department of Physics, Virginia Commonwealth University, Richmond, Virginia 23284, USA \\ ${ }^{2}$ School of Physics, Huazhong University of Science and Technology, Wuhan 430074, China
}

(Received 1 February 2013; accepted 17 July 2013; published online 8 August 2013)

\begin{abstract}
Electronic transport across a $\mathrm{FeMg}_{8}$ magnetic superatom and its dimer has been investigated using a density functional theory combined with Keldysh nonequilibrium Green's-function formalism. For a single cluster, our studies for the cluster supported in various orientations on a $\mathrm{Au}(100)$ surface show that the transport is sensitive to the contact geometry. Investigations covering the cases where the axes of $\mathrm{Mg}$ square antiprism are $45^{\circ}$, perpendicular, and parallel to the transport direction, show that the equilibrium conductance, transferred charge, and current polarizations can all change significantly with orientation. Our studies on the transport across a magnetic superatom dimer $\mathrm{FeMg}_{8}-$ $\mathrm{FeMg}_{8}$ focus on the effect of electrode contact distance and the support. The calculated $I-V$ curves show negative differential resistance behavior at larger electrode-cluster contact distances. Further, the equilibrium conductance in ferromagnetic state shows an unusually high spin polarization that is about $81.48 \%$ for specific contact distance, and a large magnetoresistance ratio exceeding $500 \%$ is also found. The results show that the superatom assemblies can provide unusual transport characteristics, and that the spin polarization and magnetoresistance can be controlled via the contact geometry. () 2013 Author(s). All article content, except where otherwise noted, is licensed under a Creative Commons Attribution 3.0 Unported License. [http://dx.doi.org/10.1063/1.4817335]
\end{abstract}

\section{INTRODUCTION}

Extensive studies have now shown that the electronic states in small symmetric metal clusters are grouped into shells that can be labeled as $1 \mathrm{~S}, 1 \mathrm{P}, 1 \mathrm{D}, \ldots$, much in the same way as in atoms. ${ }^{1,2}$ This grouping results largely from the quantum confinement of the electron gas in metal clusters. This has given rise to the conceptual framework enabling description of the stable clusters as "superatoms" with the orbitals being designated as "superorbitals." The superorbitals are generally distributed over multiple atoms and the partial filling of an electronic shell does not always lead to high spin multiplicity as in atoms. This is because the clusters can undergo structural deformations that can lower the energy by lifting the degeneracy of electronic shells. One way to stabilize superatoms with high spin multiplicity is to introduce magnetic impurities with partially filled $3 d$ shells. Here, the $3 d$ states of the impurity can hybridize with the "superatomic" D-states to induce an exchange splitting that can stabilize "magnetic superatoms." We had recently proposed FeMg 8 to be such a stable species with a spin magnetic moment of $4 \mu_{\mathrm{B}}$.

The magnetic superatoms offer a combination of the localized magnetic states arising from the orbitals containing transition metal d-component as well as delocalized $\mathrm{S}, \mathrm{P}$ states distributed over the entire cluster. One of the interesting questions is about the nature of electrical transport offered by the new entities and their assemblies. In fact, the electri-

a)Email: snkhanna@vcu.edu cal transport through molecules or clusters has attracted considerable interest both from experimental groups ${ }^{3-5}$ as well as theoretical researchers. ${ }^{6-8}$ As we recently showed, transport through magnetic superatoms ${ }^{9}$ and their assemblies can offer additional features. For example, a superatom dimer $\left(\mathrm{Cs}_{8} \mathrm{~V}\right)-\left(\mathrm{Cs}_{8} \mathrm{~V}\right)$ can act as a highly efficient spin polarizer ${ }^{10}$ leading to polarizations exceeding $80 \%$. Since alkali atoms are highly reactive, superatoms composed of less reactive elements might offer a more pragmatic system. In this regards, our recent investigations have shown that a $\mathrm{FeMg}_{8}$ cluster is not only a highly stable magnetic superatom with a magnetic moment of $4 \mu_{\mathrm{B}},{ }^{11}$ the identity of the individual motifs is maintained as the two $\mathrm{FeMg}_{8}$ clusters are assembled together.

The purpose of this paper is to investigate the electronic transport through a $\mathrm{FeMg}_{8}$ magnetic superatom and its dimer supported between two Au leads. Since the highest occupied molecular orbital (HOMO) in a cluster is spread over the outer atoms, the electronic transport is expected to be sensitive to the overlap between the connecting atoms and the leads. This suggests that it should be possible to control the transport by changing the contact geometry and separation between the cluster and the leads. The present work precisely investigates these aspects. We first consider a single superatom and investigate the effect of contact geometries, including the orientation of the cluster relative to the electrodes. We then consider a dimer and study the effect of contact geometry and distance between the cluster and the electrode. We show that the transport as well as the conducting features can be significantly controlled by changing the deposition, orientation, and the contact distance. 


\section{CALCULATION METHOD}

The theoretical investigations of the transport involved two steps. Firstly, we determined the ground state of the $\mathrm{FeMg}_{8}$ cluster by carrying out linear combination of atomicorbitals molecular orbital electronic structure calculations, and then positioned the cluster at various locations and in various orientations on a $\mathrm{Au}(100)$ surface. For each configuration, the cluster was sandwiched between two Au electrodes and was placed symmetrically on both sides. The electronic transport was then examined by using the Keldysh nonequilibrium Green's-function formalism (NEGF) within a self-consistent density functional theory (DFT). The exchange and correlation effects were incorporated using a generalized gradient approximation (GGA) functional in the form proposed by Perdew-Burke-Ernzerhof (PBE). ${ }^{12}$ Double-zeta basis sets with polarization orbitals were used to describe $\mathrm{Fe}$ and $\mathrm{Mg}$ atoms, while a single-zeta basis set was used to represent $\mathrm{Au}$ sites. The mesh cut-off energy was $150 \mathrm{Ry}$. The center integral parameter has 1024 energy points. The actual calculations of the current were carried out using the set of codes as implemented in the Atomistix ToolKit (ATK) ${ }^{13,14}$ It combines the Keldysh nonequilibrium Green'sfunction formalism with a self-consistent density-functional theory based on SIESTA code ${ }^{15,16}$ along with TranSIESTA code. ${ }^{17}$ The electronic transport properties are calculated using the fully self-consistent NEGF method as implemented in TranSIESTA code, which has previously been applied in many systems. ${ }^{18,19}$ Basically, the nonequilibrium density matrix is calculated by NEGF which satisfies the Keldysh formulation.

We were interested in the total current as well as the spin polarization. To this effect, the spin current was calculated using the non-equilibrium Green's function approach based on the Landauer-Büttike formula ${ }^{20}$

$$
I_{\sigma}=\frac{e}{h} \int{ }_{u_{L}}^{u_{R}} T_{\sigma}\left(E, V_{b}\right)\left[f_{l}\left(E-\mu_{L}\right)-f_{r}\left(E-\mu_{R}\right)\right] d E .
$$

Here $\sigma=\uparrow, \downarrow$ are the spin indices, and $\mu_{L, R}$ are the chemical potentials for left-electrode (L) and right-electrode $(\mathrm{R})$, respectively. The spin-dependent transmission coefficient $T_{\sigma}\left(E, V_{b}\right)$ depends on external bias voltage $V_{b}$ and on the spin-dependent self-consistent Kohn-Sham potentials. For a given bias voltage $V_{\mathrm{b}},\left[\mu_{L}\left(V_{b}\right), \mu_{R}\left(V_{b}\right)\right]$ corresponds to the energy region that contributes to the current integral and is usually referred to as the bias window. In the presence of external bias, the electrochemical potentials in the left/right electrode $\left(\mu_{L}\left(V_{b}\right), \mu_{R}\left(V_{b}\right)\right)$ are shifted to $\mu_{L}\left(V_{b}\right)$ $=\mu_{L}(0)+e V_{b} / 2$ and $\mu_{R}\left(V_{b}\right)=\mu_{R}(0)-e V_{b} / 2$. Here $\mu_{L}(0)$ $=\mu_{R}(0)=E_{F}$ (Fermi level) in the present calculations is set to zero, so the bias window corresponds to the range $\left[-V_{\mathrm{b}} / 2\right.$, $\left.+V_{\mathrm{b}} / 2\right] .{ }^{21,22}$ For the system at equilibrium (zero bias), the conductance $G$ was calculated via the transmission coefficients $T(E)$ at the Fermi energy $E_{\mathrm{F}}$ of the system,

$$
G=G_{0} T\left(E_{F}\right) .
$$

Here $G_{0}=\frac{2 e^{2}}{h}$, where $h$ is the Planck constant. More details about this method can be found in Refs. 14 and 17.
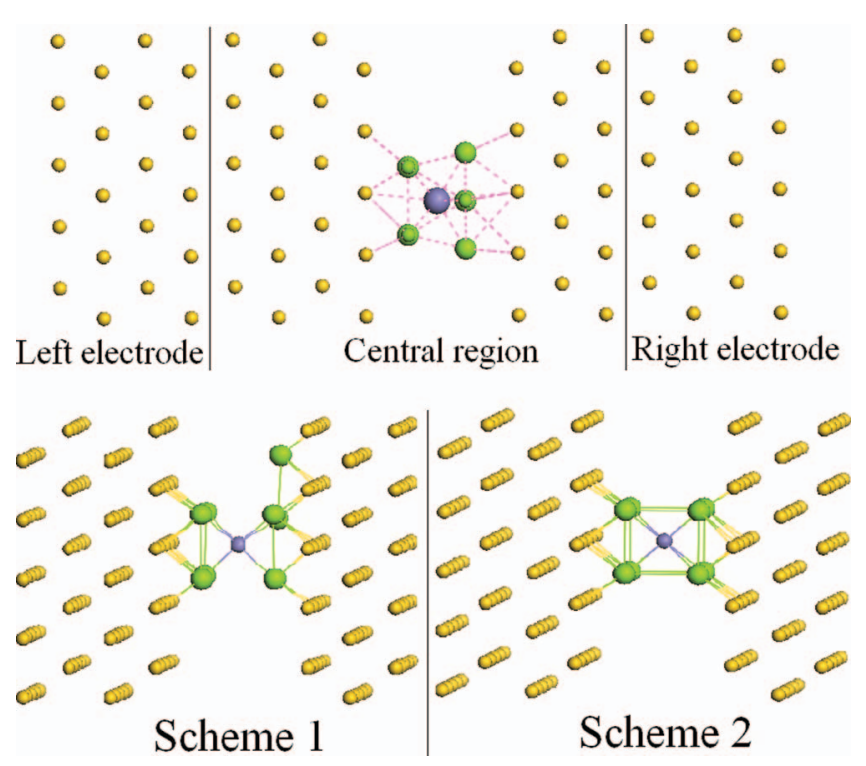

FIG. 1. The two-probe geometry of the $\mathrm{FeMg}_{8}$ superatom adsorbed on $\mathrm{Au}(100)$ surface. Blue and green circles of the superatom represent $\mathrm{Fe}$ and $\mathrm{Mg}$ atoms, respectively.

\section{RESULTS AND DISCUSSION}

In order to determine the conductance, we first considered a $\mathrm{FeMg}_{8}$ cluster supported on a $\mathrm{Au}(100)$ surface. The $\mathrm{Au}(100)$ surface was modeled by a five layer slab with a $(5 \times 5)$ unit cell as shown in Figure 1. To position the cluster on the surface, we first carried out a calculation of the near optimum location of a single $\mathrm{Mg}$ atom above the surface (in actual experiments, one can adjust the distance between the electrodes). A Mg atom was placed at a hollow site above the five-layer slab representing the $\mathrm{Au}(100)$ surface. The vertical distance between the magnesium atom and the slab was varied, and we monitored the total energy of the whole adatomslab system. The magnesium atom was allowed to relax in the direction of forces till the forces dropped below a threshold value of $0.05 \mathrm{eV} / \AA$. In the present work, we were primarily interested in calculating the transmission characteristics for various cluster surface separations, and consequently we did not relax the $\mathrm{Au}(100)$ surface in the presence of $\mathrm{Mg}$ atom or cluster. Such relaxations can affect the transport and such investigations will form the basis of future work. The lowest energy configuration corresponds to nearest $\mathrm{Mg}$-surface vertical distance of $2.07 \AA$. This separation corresponds to the nearest $\mathrm{Mg}-\mathrm{Au}$ distance $d_{\mathrm{Mg}-\mathrm{Au}}$ of $2.88 \AA$. We selected this vertical distance as the cluster-electrode contact distance for initial investigation.

To monitor the effects of relative orientation of the clusters on the spin transport properties of $\mathrm{FeMg}_{8}$ superatom, we considered three different binding configurations shown in Figure $\mathrm{S} 1$ (see the supplementary material ${ }^{24}$ ). In configuration I, shown in Figure S1(a) in the supplementary material, ${ }^{24}$ two of the $\mathrm{Mg}$ atoms occupy the bridge (near hollow) sites over the $\mathrm{Au}(100)$ surface. In configuration II, shown in Figure S1(b) in the supplementary material, ${ }^{24}$ the square antiprism has square faces perpendicular to the $\mathrm{Au}(100)$ surfaces. In configuration III, shown in Figure S1(c) in the supplementary material, ${ }^{24}$ the 
TABLE I. HOMO and LUMO energy levels at zero bias for FeMg $\mathrm{Fg}_{8}$ and its configurations in the two-probe system.

\begin{tabular}{|c|c|c|c|c|}
\hline \multirow[b]{3}{*}{ Configurations } & \multicolumn{4}{|c|}{ Molecular orbital energy $(\mathrm{eV})$} \\
\hline & \multicolumn{2}{|c|}{ Spin up } & \multicolumn{2}{|c|}{ Spin down } \\
\hline & HOMO & LUMO & HOMO & LUMO \\
\hline Isolated cluster $\mathrm{FeMg}_{8}$ & -0.57 & 0.16 & -0.30 & 0.22 \\
\hline Geometry I & -0.63 & 0.05 & -0.33 & 0.21 \\
\hline Geometry II & -0.46 & 0.14 & -0.29 & 0.30 \\
\hline Geometry III & -0.27 & 0.13 & -0.15 & 0.69 \\
\hline Optimized geometry III (Scheme 1) & -0.07 & 0.53 & -0.24 & 0.33 \\
\hline Optimized geometry III (Scheme 2) & -0.04 & 0.48 & -0.22 & 0.37 \\
\hline
\end{tabular}

square faces of the antiprism align with the $\mathrm{Au}(100)$ surfaces. For Geometry I, II, and III, the axes of Mg square antiprism is at an angle of $45^{\circ}$, perpendicular, and parallel to the transport direction, respectively. The superatom $\mathrm{FeMg}_{8}$ is assumed to be adsorbed at the $5 \times 5$ (100) direction semi-infinite gold electrodes. The periodic boundary conditions were applied in the basal plane (orthogonal to the transport direction) and a $k$-grid sampling of $3 \times 3 \times 100$ for the gold electrodes was used for determining the total energy.

A calculation of the total energy in the three configurations shows that Geometry III is the most stable configuration. Starting from this geometry, we tried to fully optimize the geometry of the cluster by relaxing all the atoms in the direction of forces till the forces dropped below a threshold value of $0.05 \mathrm{eV} / \AA$. The fully optimized cluster had one of magnesium atom move farther away from the rest of the cluster. This is shown as Scheme 1 in Figure 1. During the optimization, the four magnesium atoms on the right site of the super- atom all moved up and occupied hollow sites on the Au electrode. The four magnesium atoms originally on the left side, continue to occupy the hollow sites above the surface. Looking at this preference, we tried another starting configuration where the $8 \mathrm{Mg}$ atoms were in a cubic starting geometry. Relaxation of such a starting configuration led to a final relaxed structure where the magnesium atoms were symmetrically located on both sites. This configuration was more stable than all other configurations and is shown as Scheme 2 in Figure 1. We would like to add that in actual experiments, the geometry of the cluster can be further controlled by attaching ligands.

In order to investigate the electronic state of the isolated cluster, and its eventual change when in contact with the gold surface, we first focus on the HOMO and the lowest unoccupied molecular orbital (LUMO) energy level of isolated cluster, and its modification when the cluster is placed in contact with the gold surface. Table I lists the HOMO and LUMO of these systems at zero bias, where the Fermi level of the gold electrode has been set to be zero. In all cases, the energy gap between HOMO and LUMO of spin-up state is smaller for cluster in two-probe system than that of isolated cluster, but the reverse is the case for spin-down state. As we will show, there is also a larger density of electrons at the Fermi level for spin-up state than for the spin-down state. This can be seen through the transmission spectra and density of state (DOS), as discussed below, and it results in spin polarized currents in the two-probe system. The Au leads also result in a modification of the magnetic properties of the cluster. To this end, we calculated the Mulliken population of various atoms in the clusters and the results are tabulated in Table II. Note that there is a significant change in the magnetic moments of the cluster, as the moments are all

TABLE II. The calculated Mulliken population (in unit e) and magnetic moment (MM in unit $\mu_{\mathrm{B}}$ ) of each atom in FeMg $\mathrm{Mg}_{8}$ cluster and its configurations in the two-probe system.

\begin{tabular}{|c|c|c|c|c|c|c|c|c|c|c|c|}
\hline \multirow[b]{3}{*}{ Configurations } & & \multicolumn{9}{|c|}{ Atom } & \multirow[b]{3}{*}{ Total MM } \\
\hline & & \multicolumn{9}{|c|}{ Mulliken population } & \\
\hline & & $\mathrm{Fe}$ & $\mathrm{Mg}_{1}$ & $\mathrm{Mg}_{2}$ & $\mathrm{Mg}_{3}$ & $\mathrm{Mg}_{4}$ & $\mathrm{Mg}_{5}$ & $\mathrm{Mg}_{6}$ & $\mathrm{Mg}_{7}$ & $\mathrm{Mg}_{8}$ & \\
\hline \multirow[t]{3}{*}{ Isolated cluster } & Up & 5.304 & 1.085 & 1.087 & 1.088 & 1.087 & 1.087 & 1.089 & 1.087 & 1.088 & 4.0 \\
\hline & Down & 2.366 & 0.956 & 0.953 & 0.953 & 0.955 & 0.955 & 0.952 & 0.953 & 0.956 & \\
\hline & $\mathrm{MM}$ & 2.938 & 0.129 & 0.134 & 0.135 & 0.132 & 0.132 & 0.137 & 0.134 & 0.132 & \\
\hline \multirow{3}{*}{ Geometry I } & Up & 5.173 & 0.986 & 1.003 & 1.060 & 1.079 & 1.081 & 1.060 & 1.007 & 0.979 & 2.938 \\
\hline & Down & 2.426 & 1.079 & 1.069 & 0.968 & 0.935 & 0.928 & 0.960 & 1.053 & 1.072 & \\
\hline & MM & 2.747 & -0.093 & -0.066 & 0.092 & 0.144 & 0.153 & 0.100 & -0.046 & -0.093 & \\
\hline \multirow[t]{3}{*}{ Geometry II } & Up & 5.053 & 0.970 & 0.982 & 1.037 & 1.038 & 1.032 & 1.029 & 0.971 & 0.973 & 2.254 \\
\hline & Down & 2.511 & 1.087 & 1.094 & 0.988 & 0.985 & 0.999 & 0.997 & 1.083 & 1.086 & \\
\hline & MM & 2.542 & -0.117 & -0.112 & 0.049 & 0.053 & 0.033 & 0.032 & -0.112 & -0.113 & \\
\hline \multirow[t]{3}{*}{ Geometry III } & Up & 4.943 & 0.975 & 0.975 & 0.966 & 0.975 & 0.994 & 0.993 & 0.997 & 0.996 & 1.653 \\
\hline & Down & 2.631 & 1.059 & 1.055 & 1.048 & 1.055 & 1.079 & 1.079 & 1.078 & 1.078 & \\
\hline & MM & 2.312 & -0.084 & -0.080 & -0.082 & -0.080 & -0.085 & -0.086 & -0.081 & -0.082 & \\
\hline \multirow[t]{3}{*}{ Optimized Geometry III scheme 1} & Up & 5.357 & 1.000 & 0.998 & 1.008 & 1.006 & 0.993 & 0.984 & 0.985 & 0.992 & 2.549 \\
\hline & Down & 2.190 & 0.986 & 1.089 & 1.081 & 1.099 & 1.081 & 1.080 & 1.091 & 1.077 & \\
\hline & MM & 3.167 & 0.014 & -0.091 & -0.073 & -0.093 & -0.088 & -0.096 & -0.106 & -0.085 & \\
\hline \multirow[t]{3}{*}{ Optimized Geometry III scheme 2} & Up & 5.187 & 0.987 & 0.988 & 0.988 & 0.988 & 0.985 & 0.985 & 0.985 & 0.985 & 2.030 \\
\hline & Down & 2.315 & 1.095 & 1.095 & 1.095 & 1.094 & 1.089 & 1.089 & 1.088 & 1.088 & \\
\hline & MM & 2.872 & -0.108 & -0.107 & -0.107 & -0.106 & -0.104 & -0.104 & -0.103 & -0.103 & \\
\hline
\end{tabular}



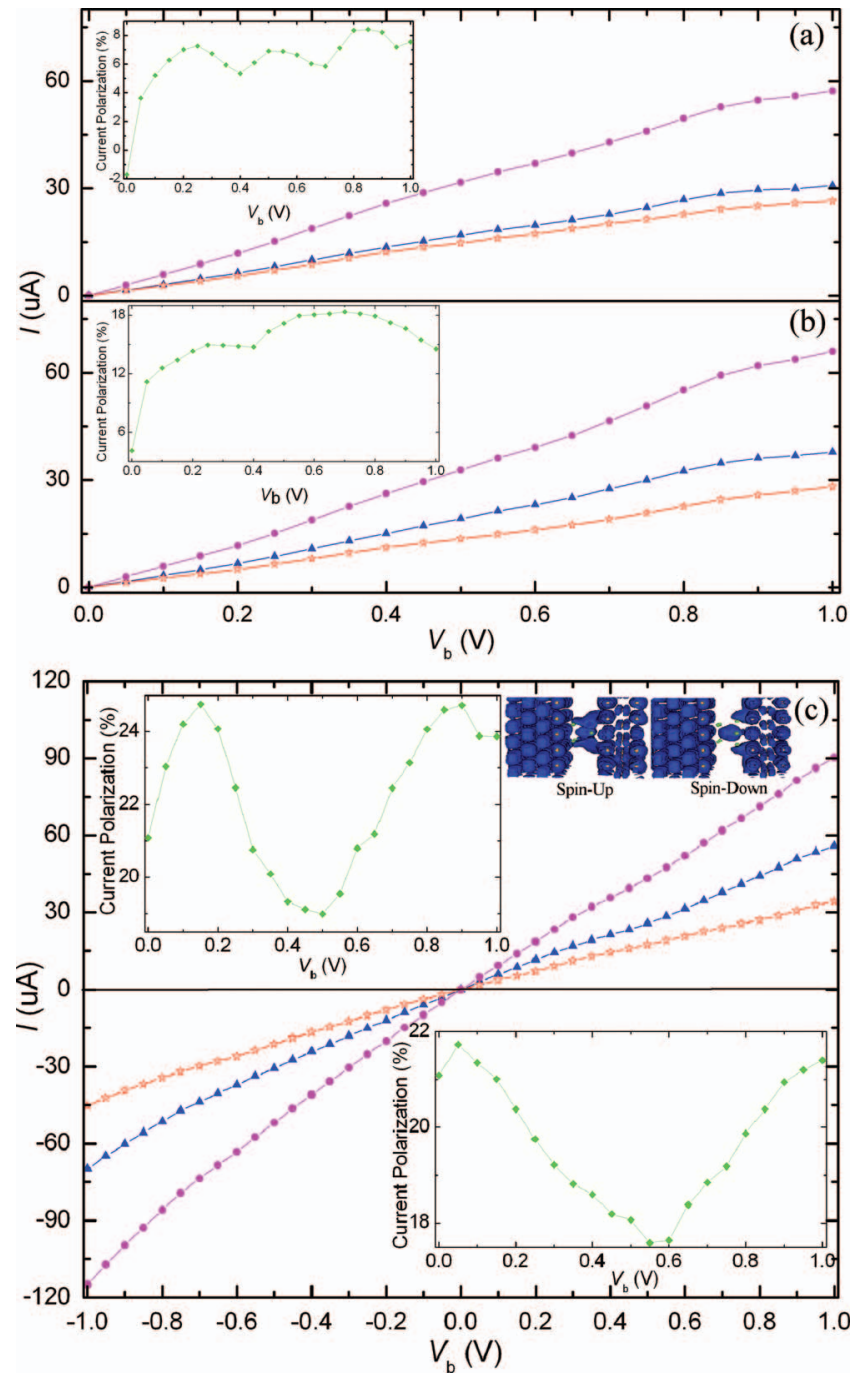

FIG. 2. Total (magenta circles), spin-up (blue triangles), and spin-down (red pentacles) $I-V$ curves for the investigated configurations: (a) Geometry I, (b) Geometry II, and (c) Geometry III and inset displays the local charge density.

smaller than those for the isolated cluster. We also found that the optimization slightly enhances the magnetic moment of clusters in Scheme 1 and 2, even though the coupling of cluster and electrode decreases the magnetic moment. Note that the magnetic moments of clusters in Scheme 1 and 2 are larger than that in Geometry III.

The calculation of the current was first carried out in Geometry I, II, and III to highlight the effect of contact geometry on the electrical properties. Since Geometry III is asymmetric, we examined the passage of current for positive and negative voltages for this case. $I-V$ curves of the three configurations of $\mathrm{FeMg}_{8}$ superatom are shown in Figure 2. The current in Geometry III is always larger than that of Geometry I and II, and the difference becomes larger with increasing bias. More specifically, the current in Geometry III is almost two times that of Geometry I at $1.0 \mathrm{~V}$. We believe that this difference is related to the number of atomic contacts, the square faces offer the maximum connectivity to the gold electrodes. For Geometry III, the difference in the connectivity of the left and right side leads to an asymmetry of the current for the positive and negative bias voltage.

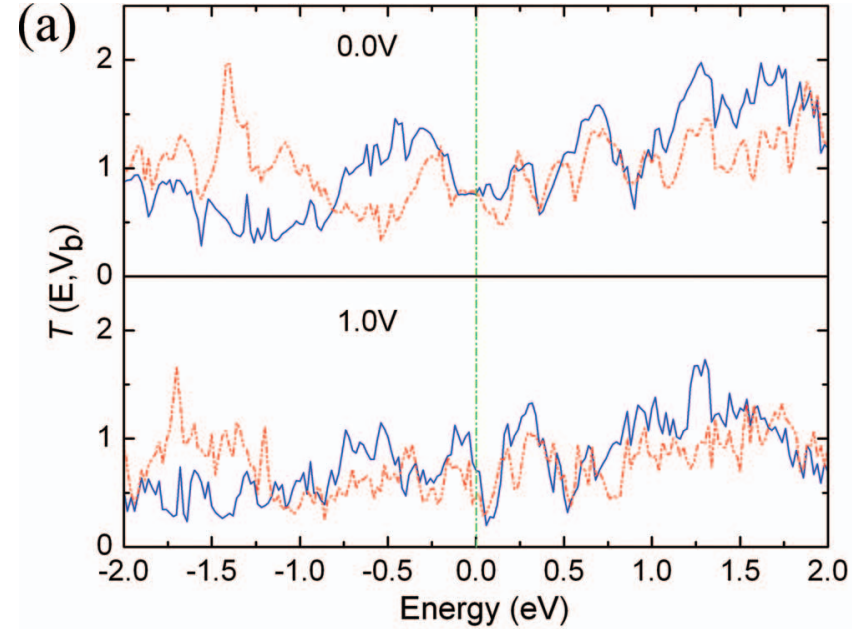

(b)
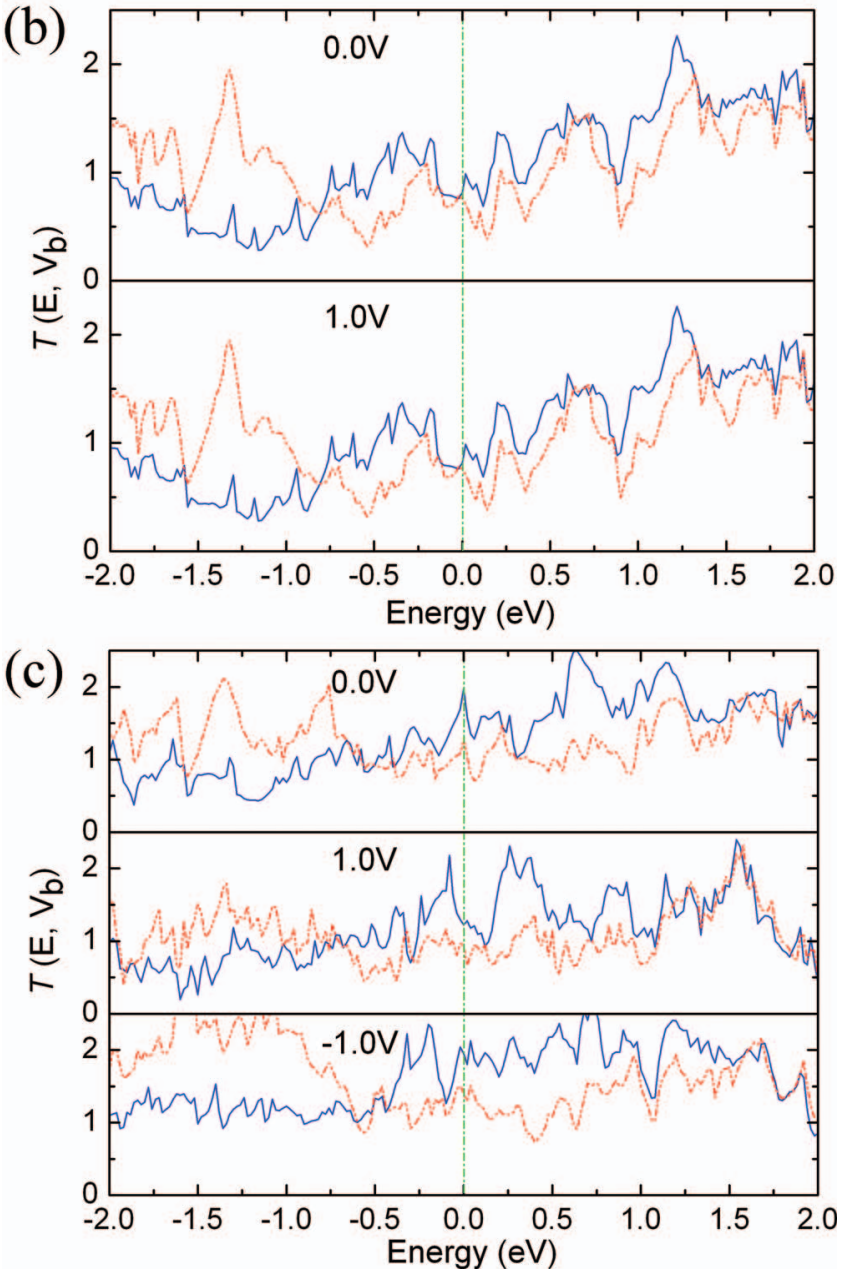

FIG. 3. The spin-polarized transmission spectra: (a) for Geometry I, (b) for Geometry II, and (c) for Geometry III. The Fermi energy is set at zero. The solid (blue) and dot (red) line denote majority and minority spin, respectively.

To further examine the origin of the effects of the relative orientation of the clusters on the spin transport properties of $\mathrm{FeMg}_{8}$, Figure 3 displays the spin-polarized transmission spectra of these three contact geometries at $0 \mathrm{~V}$ and $1.0 \mathrm{~V}$. Note that transmission coefficient of Geometry III is much larger than that of Geometry I and II near the Fermi level. Because the current $I$ is calculated from transmission spectra in the bias window by Landauer-Büttike formula (Eq. (1)), 

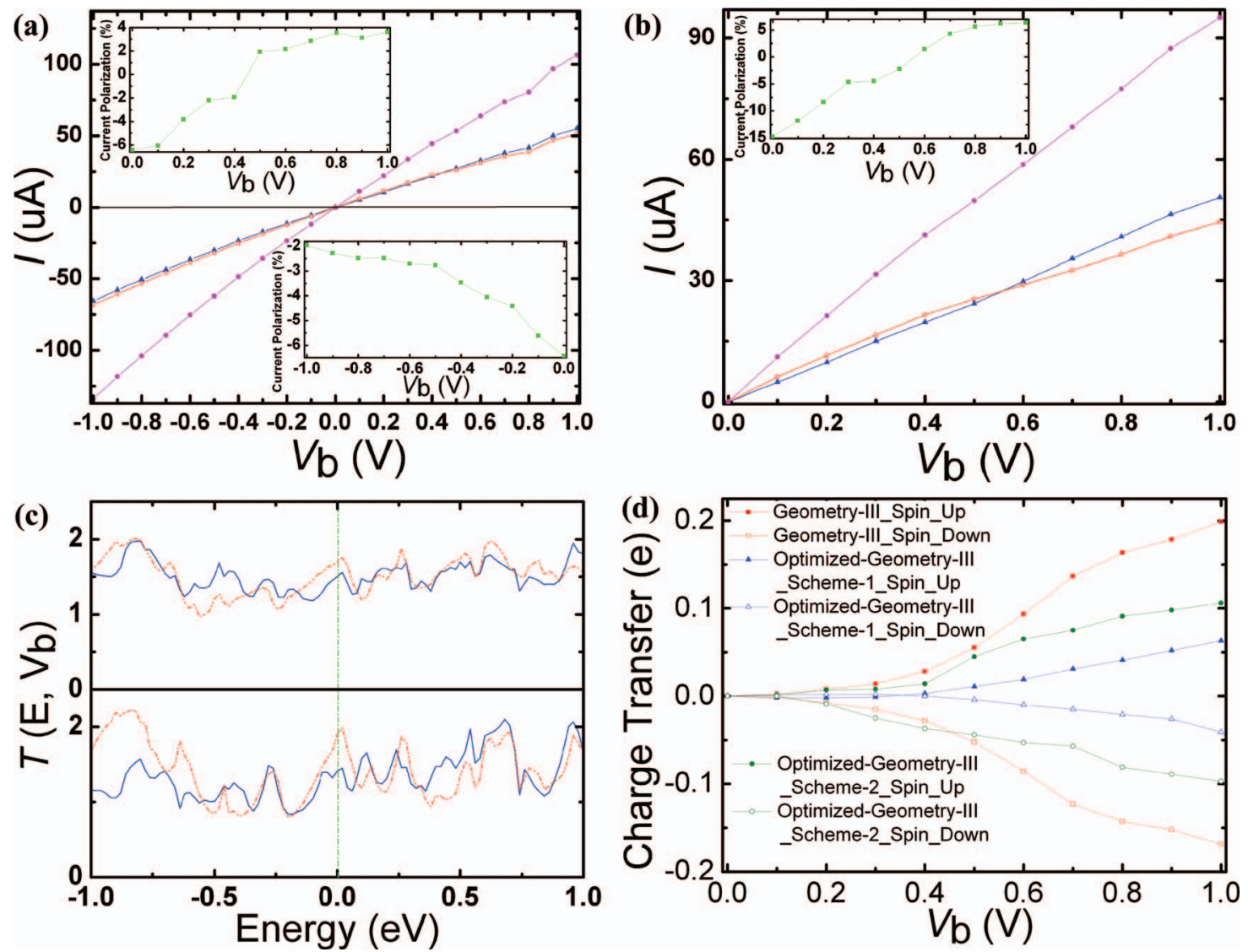

FIG. 4. Total (magenta circles), spin-up (blue triangles), and spin-down (red pentacles) $I$ - $V$ curves for the optimized Geometry III: (a) for Scheme 1 in Figure 1 , (b) for Scheme 2 in Figure 1. (c) The spin-polarized transmission spectra for Scheme 1 (top) and 2 (bottom) (the solid and dot line denote majority and minority spin, respectively). (d) Charge transfer as a function of bias for planar configuration, the positive (negative) value indicates CT from electrodes (cluster) to cluster (electrodes).

the transmission spectra of Geometry III takes much larger value than those of Geometry I and II in the energy range $[-0.5,0.5]$ at voltage $1.0 \mathrm{~V}$, thus the current of Geometry III has the largest value. To provide further insight into these differences, the DOS at zero bias for the two-probe systems was also calculated. Figure S2 in the supplementary material $^{24}$ shows the density of states and the spreading can provide an idea about how strongly the cluster couples with the electrode. ${ }^{23}$ The calculated DOS of Geometry III at Fermi level is also larger than that of Geometry I and II. It shows that Geometry III has strong coupling with electrodes through Au-Mg bond, which lets the electrons near the Fermi level easily transmit through the cluster resulting in a better transmission and larger conductance.

In addition to current, one can also calculate the spin polarization $(P)$ of the resulting current. This is shown by the inset of Figure 2, where we have defined $P$ as $P=\left(I^{\uparrow}-I_{\downarrow}\right) /\left(I^{\uparrow}\right.$ $+I_{\downarrow}$ ). The current polarization of Geometry III is also always larger than that of Geometry I or II, the largest current polarization of Geometry III is about $25 \%$ and is in the positive bias range.

In the above text, we have focused on the electrical transport in clusters deposited on the surface without relaxing the geometry. We now consider the effect of relaxation of the geometry of the cluster. As mentioned before, the completely relaxed configuration corresponds to the relaxed structures ob- tained from Geometry III shown in Figure 1. We calculated the transport properties of optimized Geometry III and the results are shown in Figures 4(a) and 4(b) for the Scheme 1 and 2 , respectively. Notice that the relaxation can have substantial effect on the current and polarization.

We have examined both the positive and negative voltages in Scheme 1 since the geometry is asymmetric and this can lead to asymmetry in current voltage characteristics. Figure 4(c) shows the transmission function and illustrates that the differences in current are associated with the changes in electronic structure that modulates the electrical transport. To provide a qualitative picture, we examine the changes in the gap. For optimized Geometry III, the spin-up HOMOLUMO gap of Scheme 1 and 2 are larger than that of Geometry III, but the spin-down HOMO-LUMO gap are smaller than that of Geometry III (shown in Table I), leading to decrease of current polarization in Scheme 1 and 2. In Figure 4(d), we show the charge transfer (CT) of the cluster in Geometry III and its optimized structure Scheme 1 and 2 under various biases. The reported values are based on a Mulliken population analysis, where we have listed the charge at each bias relative to the one at zero bias. A positive (negative) value indicates CT from electrodes (cluster) to cluster (electrodes). Note that the CT increases with the enhancement of the bias voltages for Geometry III, but for Scheme 1 and 2, because of the relaxation, smaller additional 


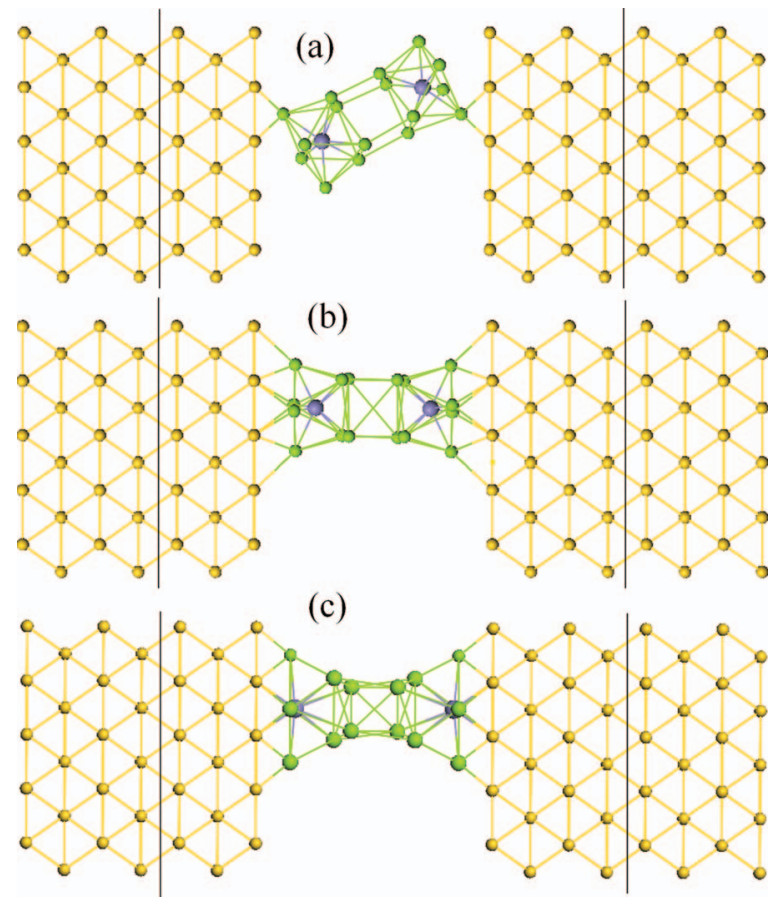

FIG. 5. Schematic plot of the two-probe system: (a) atop configuration, (b) planar configuration, and (c) fully optimized planar configuration. Blue and green circles of the superatom represent $\mathrm{Fe}$ and $\mathrm{Mg}$ atoms, respectively. The region within two black lines is the central scattering region, and the remaining parts are the left and right electrodes.

charge is drawn out of the cluster after the external bias is applied.

In the above, we have focused on a single superatomic cluster. One of the objectives of this work was to explore the properties of assemblies of such units and here we consider the transport through a dimer of $\mathrm{FeMg}_{8}$ superatoms. In a single $\mathrm{FeMg}_{8}$ superatom, the $\mathrm{Mg}$ sites form a square antiprism with an interior Fe atom. Our previous studies have shown that in a FeMg${ }_{8}-\mathrm{FeMg}_{8}$ super-molecule, the individual clusters maintain their structural identity, and the ground state of the super-molecule is an antiferromagnetic (AFM) arrangement of spin moments located at the individual clusters. The combination also possesses a ferromagnetic (FM) state only $0.18 \mathrm{eV}$ above the antiferromagnetic state. To study the transport through the dimer, we use a two-probe model as displayed in Figure 5. We have considered two different contacting geometries of the superatom dimer $\mathrm{FeMg}_{8}-\mathrm{FeMg}_{8}$ : one is atop configuration, where there is one magnesium atom of the antiprism in contact with the $\mathrm{Au}(100)$ surface (Figure 5(a)); and another is planar configuration, where there are four planar magnesium atoms of the antiprism that are in contact with $\mathrm{Au}(100)$ surface and are located at or near the hollow sites of the surface (Figure 5(b)). The superatom dimers are assumed to be adsorbed on the $5 \times 5$ (100) direction semi-infinite gold electrodes with periodic boundary conditions in the basal plane (orthogonal to the transport direction). Note that for the atop configuration in Figure 5(a), the Mg atom can occupy ontop, bridge, or hollow positions above the Au surface. Since the superatom-electrode distance is an important parameter for the transport, we first carried out total energy calculations to determine the most favorable configuration. The calculated total energy of the superatom $\left(\mathrm{FeMg}_{8}\right)_{2}$-absorbate gold slab as a function of the Mg-slab vertical distance is shown in Figure $S 3$ (see the supplementary material ${ }^{24}$ ) for all the possible configurations including atoms in (a) in an on-top, bridge, and hollow configurations, and (b) a planar configuration without full optimization of the cluster geometry, where the zero point of the energy has been chosen with respect to the optimal distance for each configuration. This figure displays that the equilibrium superatom-surface vertical distance is $2.119 \AA$ for planar configuration, and that for atop configurations it is $1.520,1.950$, and $2.319 \AA$ for hollow, bridge, and on-top sites, respectively. Through binding energy calculations, we also found that (not shown in Figure S3 in the supplementary material ${ }^{24}$ ) the most stable configuration corresponds to the superatom dimer $(\mathrm{FeMg})-\left(\mathrm{FeMg}_{8}\right)$ in a planar configuration on $\mathrm{Au}(100)$ surface, and the hollow site amongst atop configuration is the most energetically favorable. In addition to these configurations, we also fully optimized the superatom in two-probe system of planar configuration and that of hollow site in the atop configuration, finding the superatom in the hollow site strongly distorting and finally transforming to a planar configuration. In the planar configuration, because of the effect by the Au electrode, after optimization, the iron atoms in the superatom moved outside to the electrode, and almost in the same plane as $\mathrm{Mg}$ as shown in Figure 5(c).

Tables S1 and S2 in the supplementary material ${ }^{24}$ list the HOMO, the LUMO, and Mulliken population of each atom of these systems at zero bias, respectively. The qualitative behaviors of the changes of HOMO-LUMO gap (Table S1 in the supplementary material ${ }^{24}$ ) and magnetic moment (Table S2 in the supplementary material ${ }^{24}$ ) in most cases are the same as those of $\mathrm{FeMg}_{8}$. However, the optimization strongly enhances the magnetic moment of optimized $\mathrm{FeMg}_{8}$ dimer in planar configuration. The magnetic moment of isolated $\mathrm{FeMg}_{8}$ dimer is $4.0 \mu_{\mathrm{B}}$ in FM state, but that of the optimized $\mathrm{FeMg}_{8}$ dimer in planar configuration is $6.8 \mu_{\mathrm{B}}$ and the magnetic moment of each $\mathrm{Fe}$ atom is around $3.5 \mu_{\mathrm{B}}$. Following the relaxation, the $\mathrm{Fe}$ atoms in the dimer move out, and are almost in the same plane as outside $\mathrm{Mg}$ atoms (Figure 5(c)). Consequently, the interaction between the two Fe atoms is weak, each $\mathrm{FeMg}_{8}$ cluster in the dimer maintains its own character. Figure 6

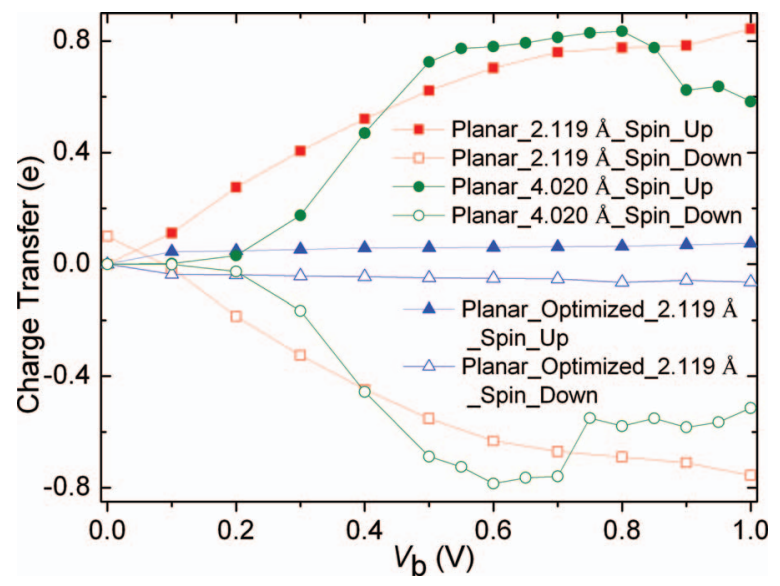

FIG. 6. Charge transfer as function of bias for planar configuration, the positive (negative) value indicates CT from electrodes (cluster) to cluster (electrodes). 

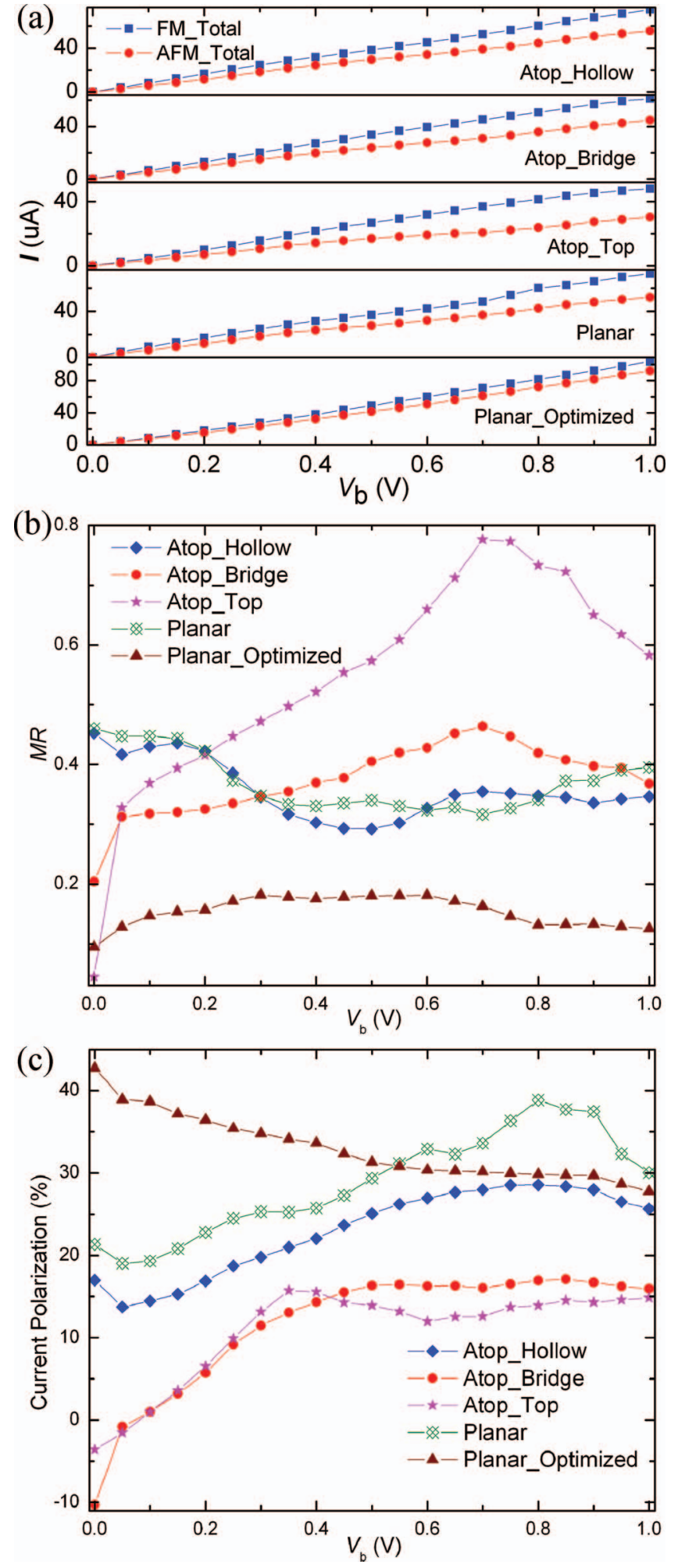

FIG. 7. (a) Total $I-V$ curves, (b) magnetoresistance (MR) ratio, and (c) current polarization for the investigated configurations at the equilibrium positions.

displays the CT of planar configuration. From Figure 6 one can find that the bias induces a rapid change in CT for planar configuration at equilibrium distance $(2.119 \AA)$, however, the CT of the optimized structure almost maintains the same value during the applied bias.
Figure 7(a) shows the calculated current-voltage $(I-V)$ curves for the equilibrium positions in planar and atop configuration for the FM and AFM configurations. The $I-V$ curves show almost linear characteristics for the whole applied bias and currents of FM state are larger than those in the AFM state. We also calculated the magnetoresistance $(\mathrm{MR})=\left[I_{\mathrm{F}}\right.$ $\left.-I_{\mathrm{AFM}}\right] / I_{\mathrm{AFM}}$, where $I_{\mathrm{F}}$ and $I_{\mathrm{AFM}}$ are the currents in the FM and AFM states at the same applied voltage, respectively. The calculated values of MR using the $I-V$ curves are shown in Figure 7(b). Near the bias of $0.0 \mathrm{~V}$, the current is represented by equilibrium conductance. At this value, we find $R_{\mathrm{MR}}$ $\sim 45.28 \%$ (hollow site), $20.43 \%$ (bridge site), and $0.05 \%$ (top site) for atop configuration and a $R_{\mathrm{MR}} \sim 46.03 \%$ for planar configuration at zero bias. When bias increases, the MR of top site for atop configuration increases quickly, and then reaches $77.62 \%$ at $0.7 \mathrm{~V}$. It is interesting to examine the amount of current polarization in the FM state. This is given in Figure 7(c), which shows that the polarization of planar configuration is larger than those of atop configurations; the largest polarization is about $39 \%$ in planar configuration. For atop configuration, the largest polarization is in the hollow site and is about $29 \%$. We also found that the optimization had little effect on the spin transport properties of the superatom, which increases the current polarization, but reduces the MR. The results also show that compared to a single unit, the cluster assembly can improve the current polarization, but decreases the current.

In practice, it may be possible to increase the separation between the electrodes. Consequently, we undertook studies where the spacing between the electrodes was increased by $0.5 \AA$ at a time. Such an increase translates into different changes in the vertical distance between $\mathrm{Mg}$ and the $\mathrm{Au}(100)$ surface depending on the location of the dimer. The spin current for the cluster-electrode vertical distance of $4.020 \AA$ (planar configuration), and for 2.067, 3.030, and $4.030 \AA$ (atop configuration) were calculated and are shown in Figure 8(a). Compared to the small cluster-electrode vertical distance (Figure 7(a)) where the currents display linear properties, the increase in the cluster-electrode vertical distance, results in Negative Differential Resistance (NDR) behavior for selected bias. A larger NDR is in the spin-up channel of FM state for atop configuration (hollow site) with $d_{\mathrm{Mg} \text {-Au(100) }}$ of $4.030 \AA$ and the spin-down channel of FM state for planar configuration, and both appear at $0.6 \mathrm{~V}$. For clarity, the enlarged figures about NDR for these cases are shown in the inset. The peak-to-valley ratio is 1.32 and 1.16 for atop and planar configuration, respectively. More interestingly, we find that the MR (Figure 8(b)) and current polarization (Figure 8(c)) all increase with the increasing cluster-electrode vertical distance. The largest MR is about $504.21 \%$ at $0.5 \mathrm{~V}$ and $507.39 \%$ at $0.55 \mathrm{~V}$ for atop (hollow site with $d_{\mathrm{Mg}-\mathrm{Au}(100)}$ of $4.030 \AA$ ) and planar configuration, respectively, indicating unusually large magnetoresistance effect. At zero bias, the current polarization for atop configuration at hollow site with $d_{\mathrm{Mg}-\mathrm{Au}(100)}$ of $4.030 \AA$ is about $81.48 \%$, which can result in an efficient spin filtration effect. However, the polarization in AFM state of all the considered geometries is very small. The above significant properties of $\mathrm{FeMg}_{8}$ superatom dimer, such as NDR with high MR and current 

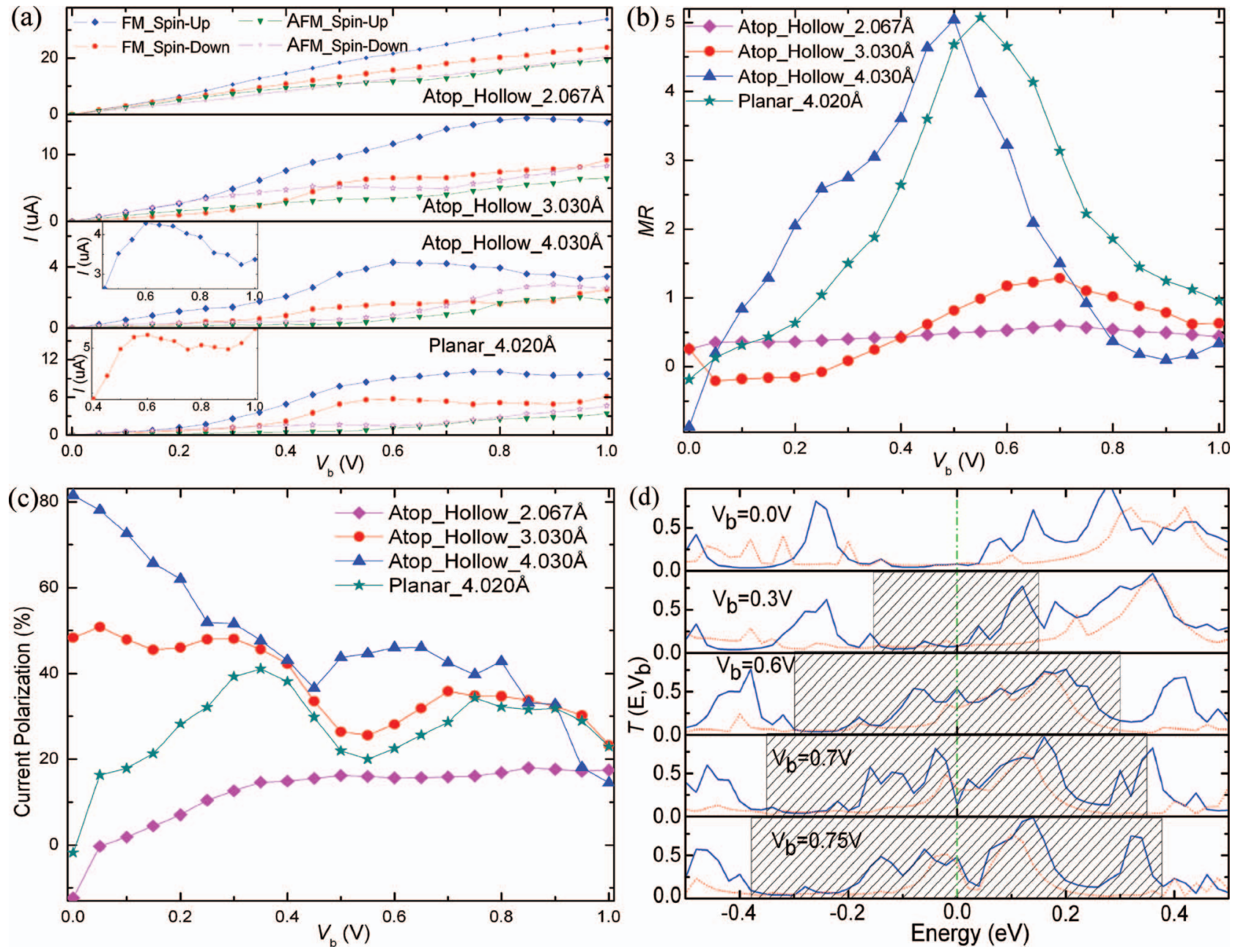

FIG. 8. (a) Spin currents, (b) magnetoresistance (MR) ratio, and (c) current polarization of the investigated configurations with larger vertical clusters-electrodes distance. (d) Transmission coefficient $T_{\sigma}\left(E, V_{b}\right)$ vs. energy $E$ for planar configuration with $d_{\mathrm{Mg}-\mathrm{Au}(100)}$ of $4.020 \AA$. (The solid and dot line denote majority and minority spin, respectively. The filled region represents the bias window.)

polarization, provide an excellent opportunity for spintronic nanodevices.

The voltage dependence of NDR and spin injection factor can be understood from the behavior of the transmission coefficient $T\left(E, V_{b}\right)$, since the current is essentially calculated by the $T\left(E, V_{b}\right)$ over the bias window through Eq. (2). Figure 8(d) and Figure S4 in the supplementary material ${ }^{24}$ show the $T\left(E, V_{b}\right)$ under several bias voltages for planar configuration with $d_{\mathrm{Mg}-\mathrm{Au}(100)}$ of $4.020 \AA$ and atop configuration at hollow site with $d_{\mathrm{Mg}-\mathrm{Au}(100)}$ of $4.030 \AA$, respectively. Due to the weak coupling of the cluster to the electrode, the biasdependent $T\left(E, V_{b}\right)$ shows several sharp peaks. The spin-up channel and the spin-down channel contribute to the $T\left(E, V_{b}\right)$ and both cross the Fermi level, which indicates that the spinup and spin-down channel conductance are through both the highest occupied molecular orbital and the lowest unoccupied molecular orbital of $\mathrm{FeMg}_{8}$ superatom dimer in the scattering region. From Figure S4 in the supplementary material ${ }^{24}$ we can find that the $T\left(E, V_{b}\right)$ is almost inhibited in spin-down channels around Fermi level, while $T\left(E, V_{b}\right)$ in the spinup channel is sharp and several orders of magnitude larger than that in spin-down channel around Fermi level. This is why there is much larger current polarization at low bias.
Figure 8(d) also shows that there is a resonance state for spin-down channel around Fermi level. As bias increases, the $T\left(E, V_{b}\right)$ moves to the low energy region, and another much larger resonance state comes to the Fermi level, resulting in rapid increase in $I \downarrow$. At the bias of $0.6 \mathrm{~V}$, this resonance state reaches maximum, then the current reaches its maximum. But when the bias continuously increases, this resonance state passes the Fermi level, which causes the decrease of the spindown conductance, leading to the NDR behavior. The NDR behavior for planar configuration with $d_{\mathrm{Mg}-\mathrm{Au}(100)}$ of $4.020 \AA$ can also be investigated by the CT (Figure 6). When bias increases, electrode gets more extra charges from the cluster, and the $\mathrm{CT}$ reaches a maximum around $0.60 \mathrm{~V}$, then decreases with the increasing bias leading to the NDR. There are similar characters of $T\left(E, V_{b}\right)$ and $\mathrm{CT}$ in atop configuration at hollow site with $d_{\mathrm{Mg}-\mathrm{Au}(100)}$ of $4.030 \AA$. Due to the geometric symmetry of the $\mathrm{FeMg}_{8}-\mathrm{FeMg}_{8}$ device, $T\left(E, V_{b}\right)$ is roughly the same for both $\sigma=\uparrow, \downarrow$ in AFM state.

\section{CONCLUSIONS}

In summary, we have carried out first-principles calculations in combination with NEGF approach to examine the 
spin-polarized transport, the effects of relative orientation of clusters on surfaces, and the effect of cluster electrode separation on the spin transport of $\mathrm{FeMg}_{8}$ magnetic superatom and its dimer. For a single cluster, we examined three different binding configurations, which are respectively named Geometry I, II, and III, where the axes of $\mathrm{Mg}$ square antiprism are $45^{\circ}$, perpendicular, and parallel to the transport direction, respectively. The results show that the equilibrium conductance, transferred charge, and current polarization of Geometry III are much larger than those of Geometry I and II which could be rationalized in terms of connectivity. The results also show that when the clusters are assembled to form a dimer, the current decreases, but the current polarization and magnetoresistance change. Our studies on a $\mathrm{FeMg}_{8}-\mathrm{FeMg}_{8}$ dimer further show a high spin polarization of about $81.48 \%$ in ferromagnetic state at larger electrode-cluster contact distance. Such high values indicate that the $\left(\mathrm{FeMg}_{8}\right)_{2}$ dimer has the potential to act as an efficient spin filter and a spin injection resource for spintronics nanodevices. In addition, the $I-V$ curves exhibit NDR and very larger magnetoresistance ratio exceeding $500 \%$, which is essential for electronic applications.

\section{ACKNOWLEDGMENTS}

We gratefully acknowledge support from U.S. Department of Energy (DOE) through Grant No. DE-FG0211ER16213.

${ }^{1}$ S. N. Khanna and P. Jena, Phys. Rev. B 51, 13705 (1995).

${ }^{2}$ A. W. Castleman and S. N. Khanna, J. Phys. Chem. C 113, 2664 (2009).

${ }^{3}$ J. J. Parks, A. R. Champagne, T. A. Costi, W. W. Shum, A. N. Pasupathy, E. Neuscamman, S. Flores-Torres, P. S. Cornaglia, A. A. Aligia, C. A. Balseiro, G. K.-L. Chan, H. D. Abruna, and D. C. Ralph, Science 328, 1370 (2010).

${ }^{4}$ E. A. Osorio, K. Moth-Poulsen, H. S. J. van der Zant, J. Paaske, P. Hedegård, K. Flensberg, J. Bendix, and T. Bjørnholm, Nano Lett. 10, 105 (2010).
${ }^{5}$ H. B. Heersche, Z. de Groot, J. A. Folk, H. S. J. van der Zant, C. Romeike, M. R. Wegewijs, L. Zobbi, D. Barreca, E. Tondello, and A. Cornia, Phys. Rev. Lett. 96, 206801 (2006).

${ }^{6}$ R. B. Pontes, A. R. Rocha, S. Sanvito, A. Fazzio, and A. J. Roque da Silva, ACS Nano 5, 795 (2011).

${ }^{7}$ V. P. Georgiev and J. E. McGrady, J. Am. Chem. Soc. 133, 12590 (2011).

${ }^{8}$ S. Sen and S. Chakrabarti, J. Am. Chem. Soc. 132, 15334 (2010).

${ }^{9}$ J. U. Reveles, P. A. Clayborne, A. C. Reber, S. N. Khanna, K. Pradhan, P. Sen, and M. R. Pederson, Nat. Chem. 1, 310 (2009).

${ }^{10}$ H. He, R. Pandey, J. U. Reveles, S. N. Khanna, and S. P. Karna, Appl. Phys. Lett. 95, 192104 (2009).

${ }^{11}$ V. M. Medel, J. U. Reveles, S. N. Khanna, V. Chauhan, P. Sen, and A. W. Castleman, Proc. Natl. Acad. Sci. U.S.A. 108, 10062 (2011).

${ }^{12}$ J. P. Perdew, K. Burke, and M. Ernzerhof, Phys. Rev. Lett. 77, 3865 (1996).

${ }^{13}$ J. M. Soler, E. Artacho, J. D. Gale, A. García, J. Unquera, P. Ordejón, and D. Sánchez-Portal, J. Phys.: Condens. Matter 14, 2745 (2002).

${ }^{14}$ J. Taylor, H. Guo, and J. Wang, Phys. Rev. B 63, 245407 (2001).

${ }^{15}$ P. Ordejon, E. Artacho, and J. M. Soler, Phys. Rev. B 53, R10441 (1996).

${ }^{16}$ D. Sánchez-Portal, P. Ordejón, E. Artacho, and J. M. Soler, Int. J. Quantum Chem. 65, 453 (1997).

${ }^{17}$ M. Brandbyge, J. L. Mozos, P. Ordejón, J. Taylor, and K. Stokbro, Phys. Rev. B 65, 165401 (2002).

${ }^{18}$ S. K. Nielsen, M. Brandbyge, K. Hansen, K. Stokbro, J. M. Van Ruitenbeek, and F. Besenbacher, Phys. Rev. Lett. 89, 066804 (2002).

${ }^{19}$ Y. J. Lee, M. Brandbyge, M. J. Puska, J. Taylor, K. Stokbro, and R. M. Nieminen, Phys. Rev. B 69, 125409 (2004).

${ }^{20}$ B. G. Wang, J. Wang, and H. Guo, J. Phys. Soc. Jpn. 70, 2645 (2001).

${ }^{21}$ J. Taylor, M. Brandbyge, and K. Stokbro, Phys. Rev. Lett. 89, 138301 (2002).

${ }^{22}$ S. Datta, W. Tian, S. Hong, R. Reifenberger, J. I. Henderson, and C. P. Kubiak, Phys. Rev. Lett. 79, 2530 (1997).

${ }^{23}$ X. Q. Shi, X. H. Zheng, Z. X. Dai, Y. Wang, and Z. Zeng, J. Phys. Chem. B 109, 3334 (2005).

${ }^{24}$ See supplementary material at http://dx.doi.org/10.1063/1.4817335 for Figure S1 which details the two-probe geometry of the FeMg $\mathrm{Mg}_{8}$ superatom adsorbed on $\mathrm{Au}(100)$ surface: (a) Geometry I, (b) Geometry II, and (c) Geometry III; Figure S2 which shows the calculated density of states: top, middle, and bottom for Geometry I, Geometry II, and Geometry III, respectively; Figure S3 which shows the binding energy of the magnetic superatom dimer $\left(\mathrm{FeMg}_{8}\right)_{2}$-absorbate gold (100) slab as a function of the $\mathrm{Mg}$-slab vertical distance; Figure S4 which displays transmission coefficient $T\left(E, V_{b}\right)$ vs. energy $\mathrm{E}$ for atop configuration at hollow site with $\mathrm{dMg}$ $\mathrm{Au}(100)$ of $4.030 \AA$. Table S1 details the HOMO and LUMO energy levels at zero bias for $\mathrm{FeMg}_{8}-\mathrm{FeMg} 8$ dimer and its configurations in the twoprobe system, and Table S2 shows the calculated Mulliken population and magnetic moment of each atom in $\mathrm{FeMg}_{8}-\mathrm{FeMg}_{8}$ dimer and its configurations in the two-probe system. 